\title{
Severe ulcerative colitis during successful pregnancy
}

\author{
C. GREENFIELD \\ M.R.C.P. \\ I. L. CRAFT \\ F.R.C.S., M.R.C.O.G.
}

\author{
R. E. POUNDER \\ M.A., M.D., M.R.C.P. \\ A. A. M. LEWIS \\ F.R.C.S.
}

Academic Departments of Medicine, Obstetrics and Gynaecology, and Surgery, Royal Free Hospital School of Medicine, London NW3 $2 Q G$

\begin{abstract}
Summary
A 31-year-old patient presented with fulminant colitis during the 27th week of her first pregnancy. Despite failed medical treatment, colectomy was delayed because of the gravid uterus. A healthy child was delivered at 32 weeks by caesarian section, but a postoperative ileus appeared to induce transient colonic dilatation. A colectomy was performed 10 days post-partum. Both mother and child survived.
\end{abstract}

KEY WORDS: ulcerative colitis, pregnancy, caesarian section. colonic motility, paralytic ileus.

\section{Introduction}

The effect of ulcerative colitis on fertility and pregnancy has been well-documented (Willoughby and Truelove, 1980; Vender and Spiro, 1982). Fertility is not altered and pregnancy is usually uneventful, especially if the patient is in remission at the time of conception. Relapses of ulcerative colitis usually (yccur in the first trimester or the puerperium (Mogardem et al., 1981) and severe colitis during pregnancy is rare (Levy, Roisman, and Teodor, 1981). This report describes the survival of a mother and child following severe colitis which developed early in the third trimester.

\section{Case report}

The patient was investigated when 25 years old for a brief episode of diarrhoea without blood or mucus. A barium enema performed at that time was reported to be normal. Her symptoms resolved spontaneously. Five years later, the patient stopped the contraceptive pill and started her first pregnancy one year later.

At 25 weeks of gestation, the patient developed diarrhoea containing blood and mucus. Two weeks later, she sought medical attention as she was opening her bowels 8 times a day with associated lower abdominal pain. She was admitted to hospital as an emergency. She appeared well and was afebrile. Her abdomen was soft and non-tender, but was distended by a gravid uterus. The foetal heart was audible. Sigmoidoscopy showed fine ulceration of the rectal mucosa, with contact haemorrhage. A rectal biopsy confirmed the clinical diagnosis of active ulcerative colitis. The haemoglobin concentration was $11.8 \mathrm{~g} / \mathrm{dl}$, white cell count $5.7 \times 10^{9} /$ litre, and the erythrocyte sedimentation rate was $103 \mathrm{~mm}$ in the first hour. The serum albumin concentration was 37 $\mathrm{g} /$ litre. Stool examination revealed neither pathogens nor Clostridium difficile toxin.

The patient was initially treated with bedrest, prednisolone enemata, and oral sulphasalazine, $1 \mathrm{~g}$ twice a day. As the patient had not improved after 4 days of treatment, she was given prednisolone $60 \mathrm{mg}$ daily by mouth, and the sulphasalazine was increased to $1 \mathrm{~g}$ three times a day. The prednisolone enemata were continued.

Fourteen days after admission, the patient's haemoglobin had fallen to $9.7 \mathrm{~g} / \mathrm{dl}$; she received two units of whole blood by transfusion. Although the patient felt well, she continued to have diarrhoea, opening her bowels 8-12 times each day. Clinical assessment of abdominal distention was made difficult by the presence of a gravid uterus. Because of the patient's lack of progress, a plain supine X-ray film of the abdomen was taken: the colon was gas-filled and empty, but there was no colonic dilatation, and the baby's development was appropriate for the duration of pregnancy, approximately 30 weeks (Fig. 1).

On the 20th day after admission, the patient developed uterine contractions and the membranes ruptured spontaneously; she was transferred to the labour ward, and received a continuous intravenous infusion of a myometrial relaxant (isoxsuprine hy- 


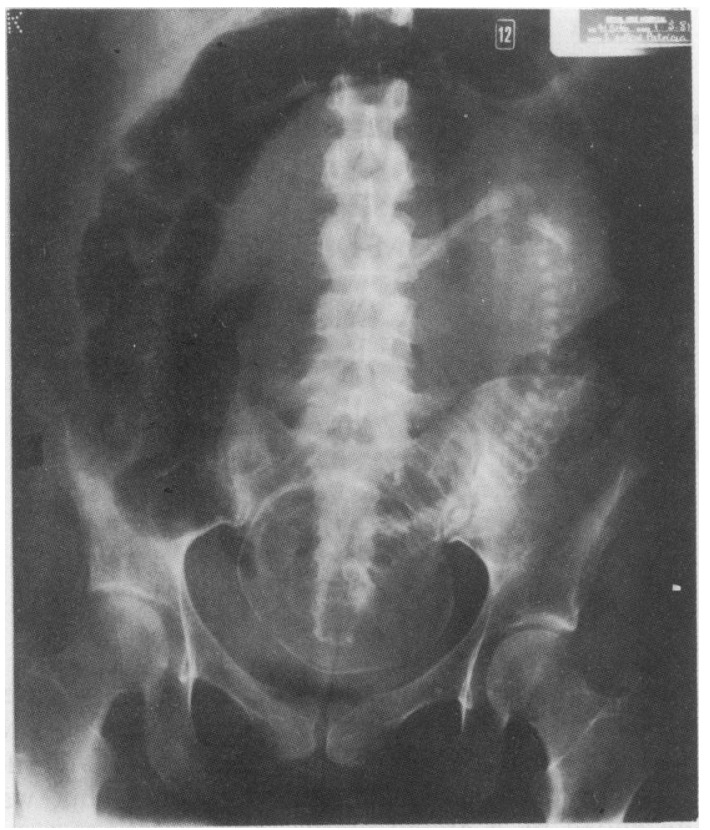

FIG. 1. Supine X-ray 14 days after admission. Gravid uterus displacing gas-filled colon, and small bowel.

drochloride $120 \mu \mathrm{g} /$ minute). The uterine contractions subsided.

Twenty-four days after admission, the haemoglobin had dropped to $9.0 \mathrm{~g} / \mathrm{dl}$, and the patient received a further transfusion of 3 units of whole blood. The patient continued to have bloody diarrhoea 8-12 times each day; sigmoidoscopy revealed an acutely inflamed rectal mucosa. The patient's colitis was still not in remission. She had been anorectic and the serum albumin had fallen to $30 \mathrm{~g}$ /litre. Parenteral nutrition was commenced and, in addition, she was changed from oral prednisolone to intravenous prednisolone $32 \mathrm{mg}$ twice a day. Metronidazole $0.5 \mathrm{~g}$ was given intravenously every $8 \mathrm{hr}$.

On the 28th day, despite the continuing isoxsuprine hydrochloride infusion, the patient entered the first stage of labour with increasing uterine contractions and a dilating cervix. The patient received intravenous dexamethasone, to protect the unborn infant from respiratory distress syndrome.

One week later, at 32 weeks of pregnancy, uterine contractions recurred and the cervix was 6-7 cm dilated. In view of the child's prematurity, a classical caesarian section was performed and a healthy $1.9 \mathrm{~kg}$ male child was delivered. At the operation, loss of haustration throughout the large bowel was noted.

Two days after the Caesarian section, the patient developed abdominal distention with absent bowel sounds (Fig. 2). On the fourth day, the patient developed a fever of $38^{\circ} \mathrm{C}$ and the abdomen was more distended and tender; sigmoidoscopy showed an inflamed rectal mucosa, without contact haemorrhage. Serial abdominal X-rays over the next 5 days showed a progressive reduction of colonic dilatation (Fig. 3), but bloody diarrhoea then returned.

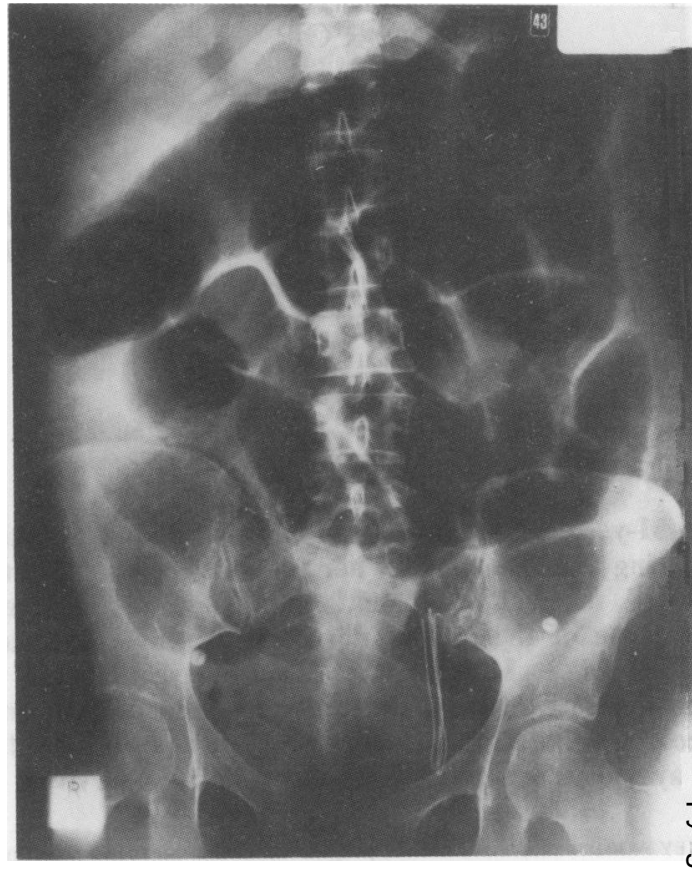

FIG. 2. Supine X-ray 2 days after Caesarian section. Dilatation of both small and large intestine.

As the colitis appeared to be deteriorating, 9 days after the caesarian section, the patient had a sub-total colectomy with ileostomy; the rectum was retained. At operation, an inflamed large bowel was observed with the most severe disease in the caecum and ascending colon. Histology showed an active colitis with numerous fissures passing to the muscularis propria, but no granulomata were present.

There was an intense polymorph and plasma cell infiltration of the mucosa and submucosa. The overall appearances were those of acute ulcerative colitis.

The patient made a rapid postoperative recovery. $\frac{D}{2}$ The rectal mucosa was initially inflamed, but responded to treatment with sulphasalazine enemata. $N$ Nine months after the colectomy, an ileo-rectal $N$ anastomosis was performed. The child has thrived, N achieving all normal milestones to 9 months.

\section{Discussion}

The patient presented in the third trimester of $\stackrel{+}{?}$ pregnancy with acute colitis. The patient's illness was 


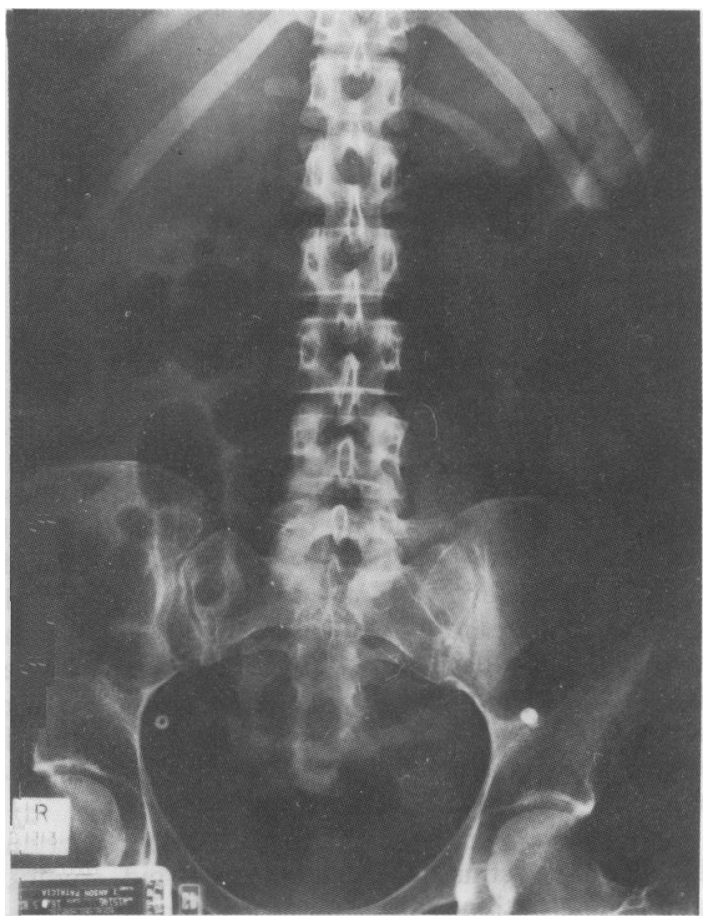

FlG. 3. Supine X-ray 10 days after Caesarian section. The whole of the colon is gas-filled, without dilatation.

difficult to assess as the gravid uterus not only made physical examination difficult, but also denied the opportunity to perform repeated plain X-rays of the abdomen. An elevated erythrocyte sedimentation rate, relative anaemia and a low serum albumin are all normal findings during pregnancy. Despite maximal medical treatment, the patient's colitis gradually deteriorated. Had she not been pregnant, it is likely that she would have had an emergency colectomy approximately one month after admission to hospital. However, the uterus containing a 30-week pregnancy would have made a colectomy extremely difficult. The pregnancy was too late to terminate, yet the baby was too immature to allow early induction. Despite early spontaneous rupture of the membranes and premature labour, delivery of the child was successfully delayed until 32 weeks gestation.

After the caesarian section, the patient developed an ileus and the colon became progressively more dilated. At the time, it was uncertain whether the colonic dilatation was due to a deterioration of the ulcerative colitis, or was a motility problem following the laparotomy and caesarian section (Wilson, 1975; Woods et al., 1978). In retrospect, it was probably the latter as the dilatation resolved to be followed by a return of bloody diarrhoea.

Severe colitis during pregnancy is extremely unusual and its presence at the beginning of the third trimester poses major management problems if both mother and child are to survive. There can be few patients who have had a laparotomy, and subsequent postoperative ileus, during an acute episode of ulcerative colitis.

\section{References}

LEVy, N., RoIsMan, I. \& TeOdor, I. (1981) Ulcerative colitis and pregnancy in Israel. Diseases of the Colon and Rectum, 24, 351.

Mogardem, M., Dobbins, W.O., Korelitz, B.I. \& Ahmed, S.W. (1981) Pregnancy in inflammatory bowel disease: effect of sulphasalazine and corticosteroids on fetal outcome. Gastroentero$\log y, 80,70$.

VENDER, R.J. \& SPIRO, H.M. (1982) Inflammatory bowel disease and pregnancy. Journal of Clinical Gastroenterology, 4, 231.

WilloughBy, C.P. \& TRUELOVE, S.C. (1980) Ulcerative colitis and pregnancy. Gut, 21, 469.

WILSON, J.P. (1975) Postoperative mobility of the large intestine in man. Gut, 16, 689.

Woods, J.H., ERICKSON, L.W., Condon, R.E., SChulte, W.J. \& SILLIN, L.F. (1978) Post operative ileus: a colonic problem? Surgery, 84, 527.

(Accepted 21 October 1982) 